\title{
Augmented Reality Applied to Building Assessment with Building Information Model Visualization
}

\author{
Márcia Regina de Freitas and Regina Coeli Ruschel \\ School of Civil Engineering, University of Campinas, São Paulo 13083-952, Brazil
}

\begin{abstract}
AR (augmented reality) is a technology that adds information to the real world adding virtual elements to its visualization in real time. AR used in AECO (architectural, engineering, construction and operations) can contribute in augmenting visualization during design, construction and operation of the buildings. This article presents a study that applies AR to building assessment with BIM (building information) model visualization. The use of AR on existing applications for smart phones and tablets is validated. AR proposed an adaptation of the method of POE (post-occupancy evaluation) subsidized. Traditional POE process model involves three phases: planning, conducting and applying. In order to incorporate AR, it is proposed a total restructuring of the planning phase, developing the research instruments in three steps: 3D modeling, model treatment and AR application development. It was observed that for POE studies, the 3D models are in large scale and need to be detailed for precise comparison. BIM models for facility management, representing building use situation, are of the highest level of detail. A balanced point between simplicity and representativeness was the solution adopted in this experiment for uploading and downloading performance issues. This article presents and discusses findings for the new proposition for the activity of research instruments development for the planning phase of POE with $\mathrm{AR}$ as well as initial tests with first results and difficulties faced.
\end{abstract}

Key words: Construction management, assessment, augmented reality, building information model.

\section{Introduction}

AR (augmented reality) can be summarized as a technology that uses VR (virtual reality) to add virtual objects to real environments in real-time, using technological devices for the visual overlapping of images $[1,2]$. Virtual reality is known as a technology that adds the dimensions of immersion and interactivity to three-dimensional computer generated models and offers an exploration that is not viable with the traditional form of representation [3]. In architecture and construction, these technologies can assist in the design and visualization of architectural forms, results of simulation and analysis of energy issues as well as in the manufacturing of structural components or directly in the construction, for example, at the construction site or during POE (post-occupancy evaluation) [4].

Corresponding author: Regina Coeli Ruschel, Ph.D., civil engineer, research fields: information and communication technology applied to building construction. E-mail: ruschel@fec.unicamp.br.
AR is in constant evolution and has been facilitated with the development of portable equipments, which allow the visualization of virtual objects in real time in mobile manner. Another improvement is the increased availability of programs for the development of AR applications.

Within this context, mobile AR can support agents responsible for technical reviews of buildings performing POE. AR may allow more accurate perception of the interventions made by the residents, collaborating with the adjustment of future developments. POE is a systematic and rigorous process to evaluate the built environment after it has been occupied for some time [5]. This process, in addition to identifying user satisfaction, building performance and pathologies, can also reveal building retrofits made to adapt to the users' requirements or change of function. Retrofits done by residents reveal important aspects and values that may be incorporated into the design process of new buildings of the same 
type. Traditional data collection for retrofits detection is made with questionnaires which depend on user memory and disciplined observation by the researcher of the built environment in comparison to original design. Therefore, if POE for retrofit detection is less based in users' memory and more based on accurate observation, this could represent more efficient results. Thus, AR may represent this gain by applying its resources for identifying reforms. The BIM (building information) model visualized against the real constructed and used building could be used in this situation. It is a promising solution to improve the state of the art in visualization in architecture, design process, construction processes and facility management, emphasizing on POE.

This article presents the use of mobile AR system for tablets supporting POE. The long-term aim is to improve retrofit identification while walking through the existing built environment. A research is presented where POE instruments incorporate AR for low income housing. The study is developed in the city of Campinas, in the state of São Paulo, Brazil.

\section{Groundings}

\subsection{Building Assessment of Low Incoming Housing}

The focus of POE should be on the occupants of the buildings and their needs, in order to give evaluators the perception of the consequences of decisions taken during the design and its results in terms of performance and attendance to user needs [5]. The main objective of this type of study is to provide knowledge based on real facts that allows the design of more suitable buildings in future projects.

In terms of dwellings designed and constructed for the low-income population, that is social housing, it is noted that the projects do not always meet users' expectations. This fact in Brazil, along with the lack of urban infrastructure, creates dissatisfaction and causes structural, functional or aesthetic user interventions in housing in the search for a better quality of life [6]. POE collected data are in the form of numbers, text, images, drawing and recordings. The visualization of results is usually indirect in the form of tables, graphs and schemas. Therefore, the use of technological resources to facilitate design feedback can mean a step forward in the search for improvement of future construction, using such information.

In this sense, the use of AR can make the process of POE faster and better understood by technical agents that make the evaluation of housing, allowing also the improvement of the presentation. The visualization of that was designed and built in opposition to what was desired and changed by the user (the reforms made) and is what makes this technology feasible for improvements in the identification and registration of retrofits in POE. Thus, the result ceases to be text only, becoming visual and comprehensive. This research aims to associate a new technology to the process that is already established, however not widely used by professionals from the architecture and construction.

\subsection{Mobile Augmented Reality}

With although smaller but more powerful computers, new mobile applications emerge combining technologies such as global tracking, wireless communication and location-based services, allowing AR possible to be used by anyone and anywhere [7]. In early proposition of mobile AR [8], computers were carried by users using a see-through HMD (head-mounted display), allowing to move freely and with correct location through the technology of GPS (global positioning system).

With the rise of tablets and smartphones equipped with GPS, wireless or mobile communication, AR becomes more versatile [9]. These devices combine the processing power, memory, interaction technology, GPS, digital compass, and integrated camera that allow viewing of built environment and with real-time mixing of virtual objects [10]. With the ability of these mobile devices and simplification in terms of hardware, there is a possible increase in the range of potential uses for AR [11]. AR applications have been developed 
for tablets and smartphones for multiple uses: entertainment, guides and education are some examples. These applications apply constantly evolving technology, especially in the case of applications developed for use over the Internet.

\subsection{Building Information Modeling}

According to Succar [12], BIM is an emerging technology and requires a procedural change for the sectors of AECO (architecture, engineering, construction and operations), as a modeling technology and associated processes to produce, analyze and communicate information with information models of buildings [13]. Since the building information model must be reused by different agents in different situation throughout the building life cycle, these agents may input the information model in varying computer programs. This results in model transformation, therefore, interoperability is essential. Interoperability allows improved collaboration and is achieved when there is no or low loss of information from the reuse of the information model.

The evolution of a BIM model is described in the LoD (level of detail) from the lowest level in the conceptual phase, gradually increasing in level of accuracy and representation for the construction phase, manufacture and operation [14].

LoD also corresponds to level of development establishing the requirements for model content at five progressive levels of development, and the authorized uses of the model content at each level of development [15]. According to the AIA (American Institute of
Architects) Document G202-2013, these levels of development are LoD 100 for overall building massing, LoD 200 for generalized systems or assemblies, LoD 300 for specific assemblies accurate in terms of quantity, size, shape, location and orientation, LoD 400 for specific assemblies with complete fabrication, assembly, and detailing information and LoD 500 for constructed actual assemblies (as-built conditions). The model is suitable for maintenance and operations of the facility. There is a great interest in research related to the use of information models, but few investigate the effort required to generate the model in different LoDs and their impact on design [16].

LoD of BIM models affects use in mobile AR because $3 \mathrm{D}$ content must be uploaded and rendered in real time though the Internet and BIM model's LoD interfere in the model file size, consequently, in the data transmission on the web. The simplification of 3D content for uploading and downloading performance issues is required in this case and a balanced point between simplicity and representativeness should be found for the model. The LoD of the model itself may not change, but it has to be filtered to determine the final file size.

\section{Research Method}

The POE process model proposed by Preiser, Rabinowitz and White [5] is adopted, involving three phases: planning, conducting and applying (Fig. 1). In order to incorporate $\mathrm{AR}$, a total restructuring of one activity of the planning phase is proposed, that is, the development of research instruments. It is foreseen that

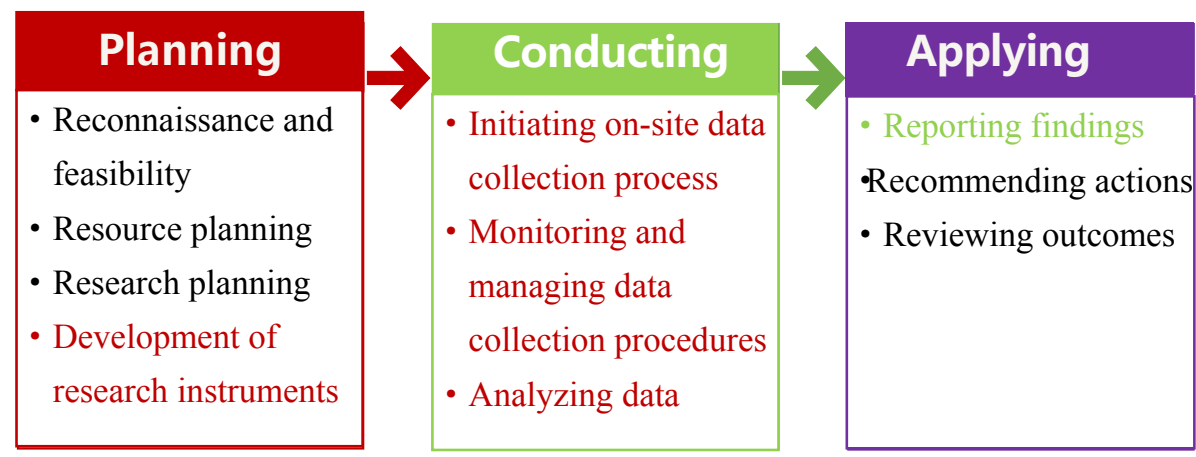

Fig. 1 POE process model proposed: activities which will be adapted (in red) and used for analysis (in green). 
other activities proposed by Presier, Rabinowitz and White [5] for this initial planning phase not change due to the use of AR, therefore are maintained. The second phase of a POE is the conducting phase, which includes on-site data collection process, monitoring and managing data collection and finally data analysis. Since instruments for data collection are totally different, this phase needs total rearrangement.

In order to evaluate the effectiveness of the use of AR for retrofit identification, two POE studies are been developed and will be compared. The study is under developed for an existing social housing development (Campinas-F of the Company of Housing and Urban Development of São Paulo) in the city of Campinas, São Paulo, Brazil (Fig. 2). The studies will differ in the following manner: One will be developed with the traditional process model proposed by Presier, Rabinowitz and White [5] and the other will be developed with this process model adapted to the use of AR (Fig. 3). Report's findings extracted from the applying phase of these studies will be compared.

This article presents and discusses findings for the new proposition for the activity of research instruments development for the planning phase of POE with AR
(Fig. 3). This activity is proposed to be developed in three steps: 3D modeling, model treatment and AR application development. 3D modeling starts from 2D

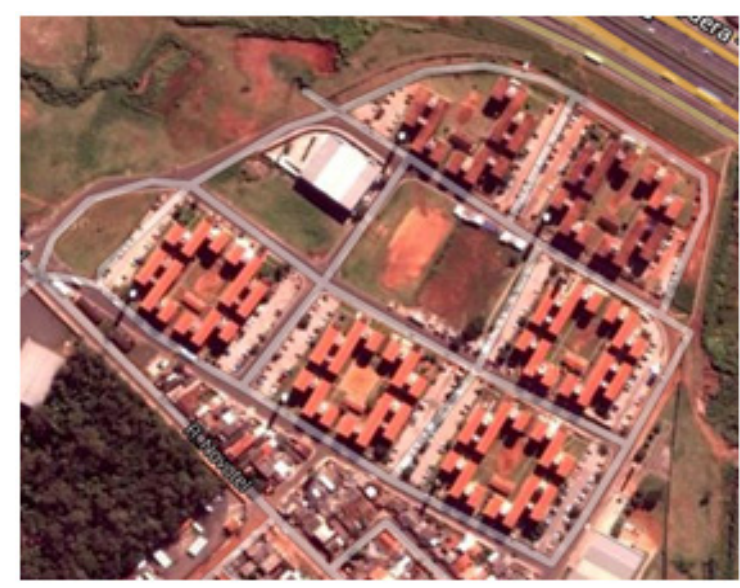

(a)

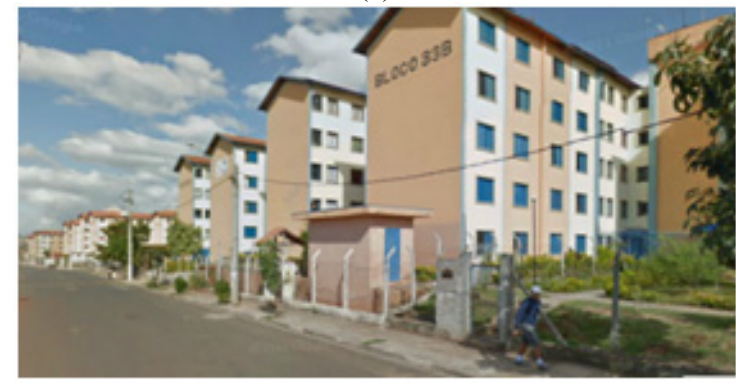

(b)

Fig. 2 Different view of the social housing: (a) aerial; (b) street.

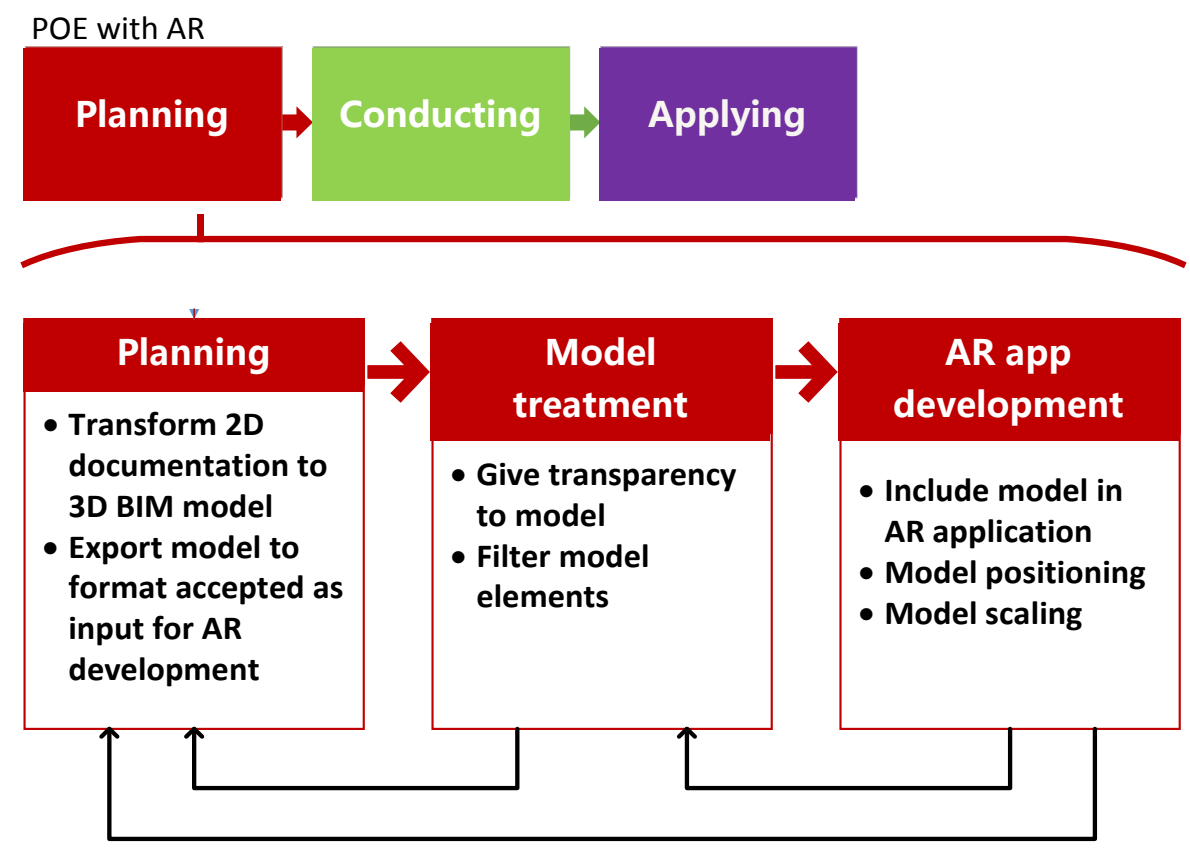

Fig. 3 Detailing of transformation of the activity of research instrument development in the planning phase of POE with AR. 
drawing because the design of the social housing in study was developed using this kind of representation. A BIM model is developed and exported to a data format accepted as input in application available for AR development. The model must be treated in order to obtain transparency and therefore allow visualization of superimposed objects (real $\mathrm{x}$ virtual). The model must also be treated in order to become lighter and simplified, to enable its use and manipulation in selected mobile AR application. Finally, the treated model is incorporated in the mobile AR application, instantiated for the site in study, requiring model positioning related to the site and scaling.

\section{Development}

The results of the activity of research instrument development in an AR application are associated to a specific built environment. This application is downloaded, installed in a mobile device and used in site by the person in charge of data collecting. The data collecting comprehends two possible situations: searching for user intervention (retrofits) on open space (outside and around the buildings) and in closed spaces (an apartment for example). Mobile AR application was developed for the open space situation using georeferenced AR and the Junaio browser.

With the ease offered by devices with multiple functionalities associated (for example georeferencing and compass), as the iPad 2 tablet (Apple, Inc.), an AR application was chosen considering the visualization of 3D models in real scale to be seen with this device. The application Junaio (Metaio Inc.) was used for the first tests. This application enables RA with or without use of markers, 3D object tracking and the ability to embed multimedia content. The 3D model imported by Junaio are OBJ (object files) or MD2. Object files are text based files describing a geometry and its texture, it supports both polygonal and free-form geometry (curves and surfaces). The MD2 format is a binary format originally created to be used in a game (Quake-2). Its characteristics includes geometric data model (triangles), animations frame by frame and structured data model.

\subsection{Modeling}

The BIM model was developed using Autodesk Revit. Terrains (natural and leveled) were modeled based on level curves and the buildings were modeled based on 2D plans and sections. Information was taken from construction drawings. However, the resulting BIM model was detailed to the level of LoD 300 (Fig. 4).

\subsection{Model Treatment}

One reason to treat the model was the need of visibility of overlapped objects (real $\mathrm{x}$ virtual), in order not to hide extra existing built environment elements when not present in the virtual model. The virtual model should represent the initial site condition. Two applications were first tested for model treatment: Autodesk 3DsMax and Blender. The export and import process from Revit to these applications resulted in loss of model information or presented difficult use, therefore were abandoned. Finally, Sketchup (Trimble) was used presenting satisfactory performance (Fig. 5).

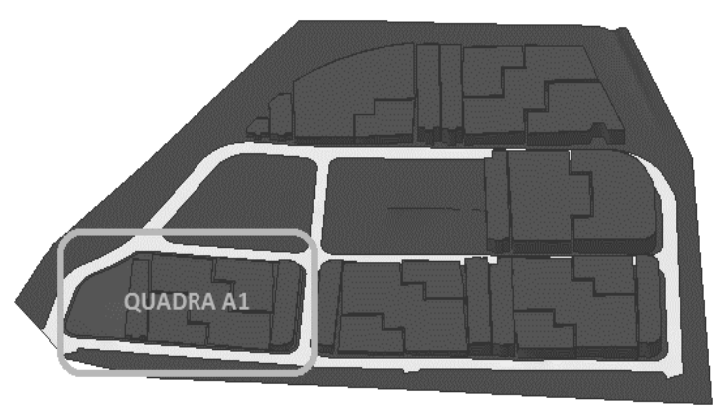

(a)

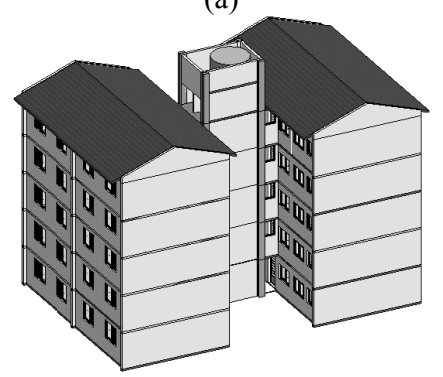

(b)

Fig. 4 BIM Revit model: (a) terrain leveled plateaus; (b) building. 


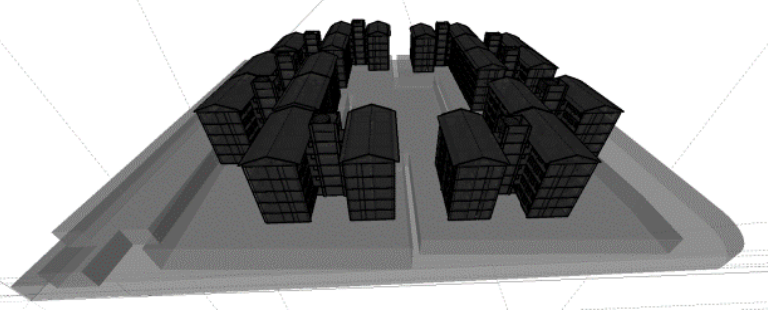

Fig. 5 One block leveled terrain and buildings with transparency (realized with Sketchup).

The low or non interoperability between used applications (modeling and AR) is a drawback and indicates need of collaboration between software development company. This limitation was partially resolved.

The model used in AR by Junaio must be uploaded to a model server and communicated to the application by internet during visualization. Therefore, there is model size limitation of 750 Kbytes. This fact does not prevent the use of larger files, however, using larger files will likely result in problems such as slow transfer increases due to internet bandwidth. This is another reason why model treatment was necessary to reduce model size. Fig. 6 shows the buildings on terrain as in the real site and presents the simplified model, i.e., the terrain with fewer buildings to obtain a file size as required by Junaio, both with transparency and simplified in terms of elements.

To visualize the $3 \mathrm{D}$ model without losing the reference or the real building, the material was modified to translucent material with an opacity about $80 \%$. Furthermore, the model was simplified by eliminating component details from doors and windows and some buildings were removed of the terrain resulting in the file size of 600 Kbytes.

\subsection{AR Application Development}

Metaio is an augmented reality company that offers products and a variety of development features. Junaio is Metaio's mobile augmented reality platform that allows the creation of content channels for the real world. It offers three platform-specific programming interfaces: Android, iOS and Windows. The programming interface for Windows offers as alternatives: the developing environment SDK (software development kit) for Windows, the $\mathrm{C}++$ language and the AREL (augmented reality experience language). Junaio mobile AR platform combines real world medium and virtual content. The accepted real world medium is 2D object tracking and detection, 3D object tracking and detection, marker tracking and detection, SLAM (simultaneous localization and mapping) tracking, instant tracking and GPS or inertial tracking. Possible virtual content are games, 2D or 3D content. The AR experience can be delivered by native app or AREL/HTML5 (HyperText Markup Language 5).

Once the virtual content is developed, it must be associated to the real world medium and delivered for use through the Junaio browser. Metaio offers various developing alternatives by means of a robust architecture and delivery system (Fig. 7). Our study used a subset of this structure, that is, the Junaio mobile AR browser for iOS interface, the programming interface for Windows platform with AREL, the Eclipse environment for PHP (open source general-purpose scripting language) development tool, WampServer for local content manage, Metaio Cloud and Junaio API (application programming interface) (Fig. 7 marked with dashed lines). The world medium was GPS or inertial tracking and the virtual content was $3 \mathrm{D}$ built environment model (described in the previous section).

The 3D content, reduced to one block of the social housing, was modeled in conditions (format, size and units - $\mathrm{mm}$ ) for the Junaio mobile AR browser, then testing regarding mobile AR creation and delivery was

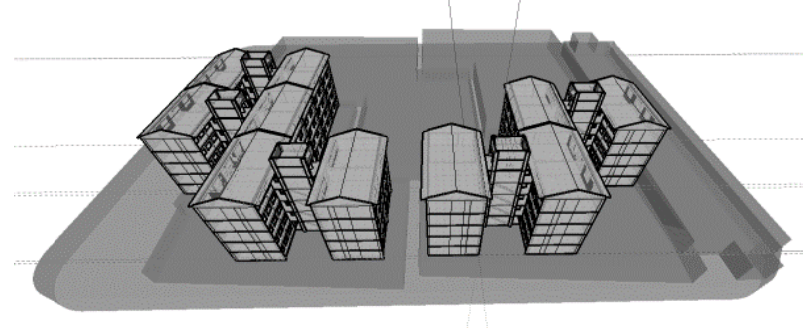

Fig. 6 Reduced and simplified model to meet file size limitation imposed by Junaio using Sketchup. 


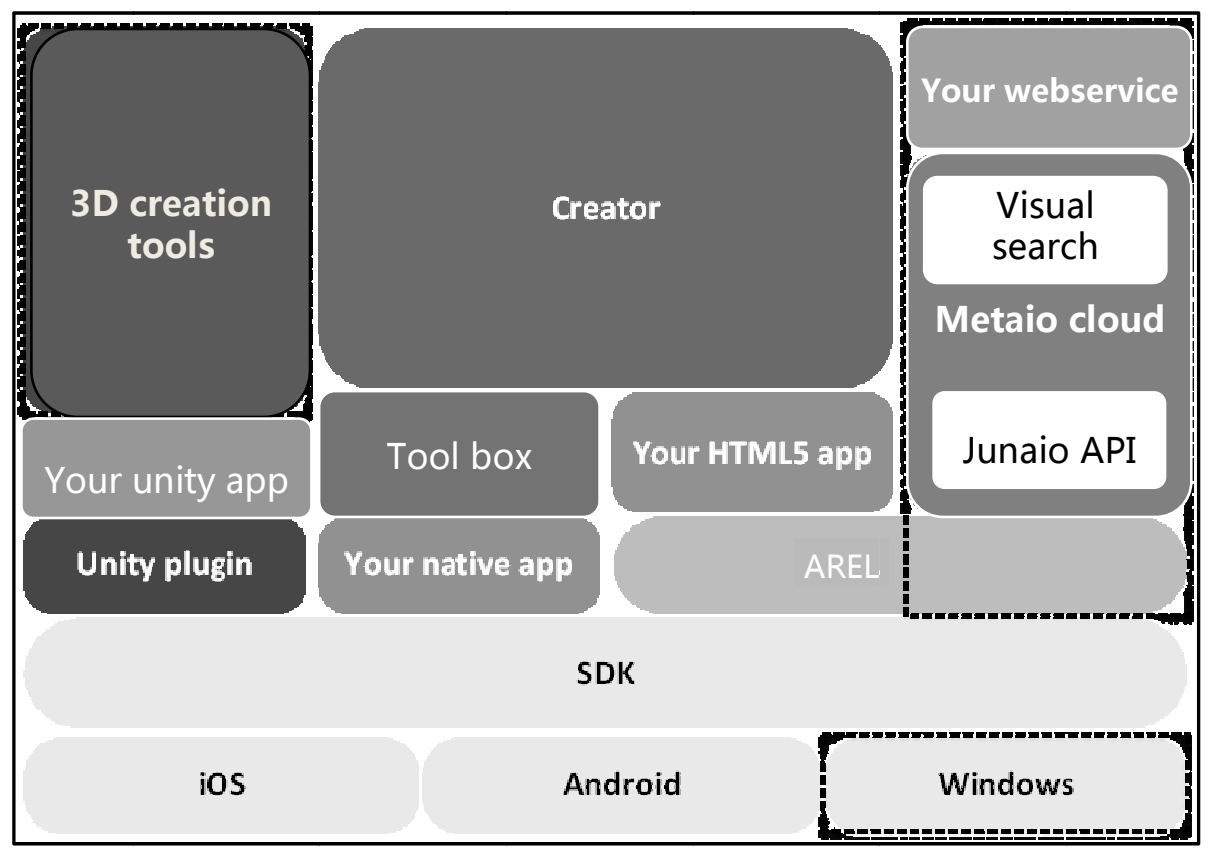

(a)

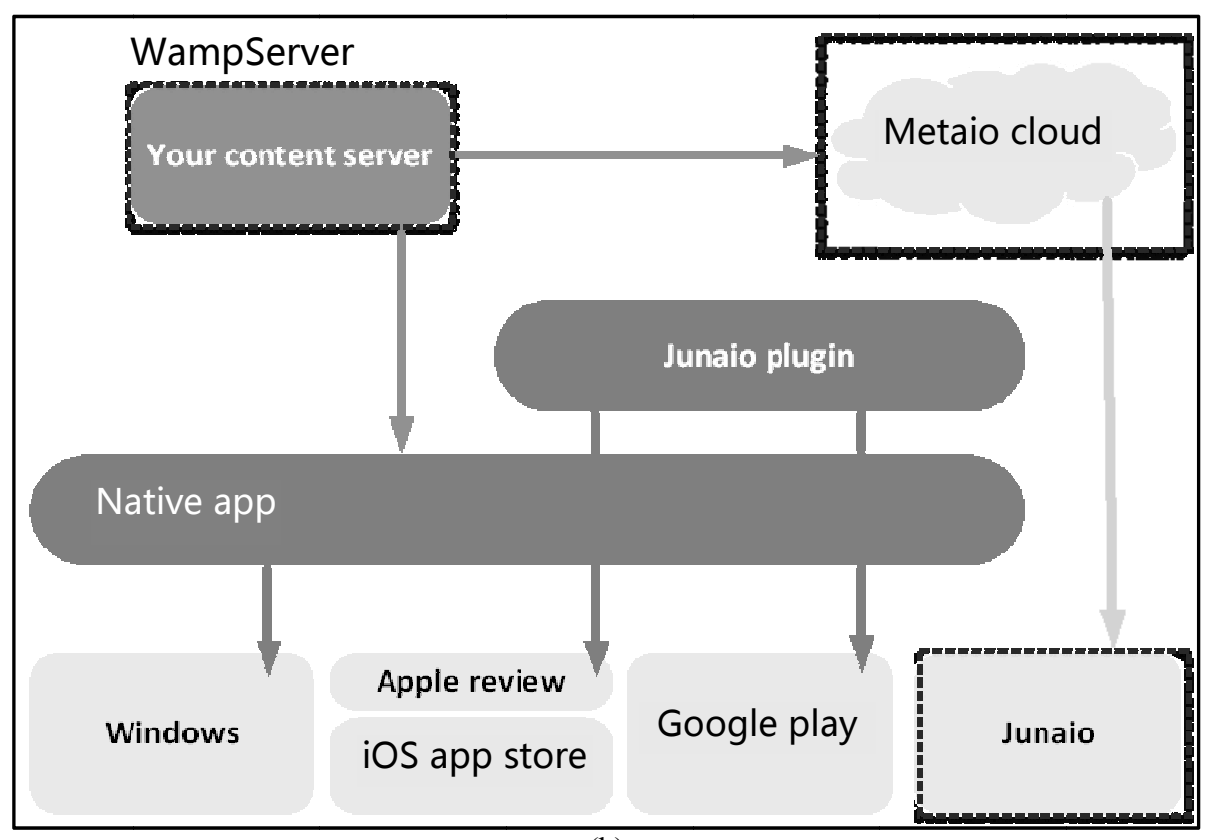

(b)

Fig. 7 Metaio resources used: (a) architecture; (b) delivery.

initiated. The mobile AR creation was realized with the Eclipse environment: this includes configuring a scene to Junaio channel. The scene is configured associating a position in the $3 \mathrm{D}$ content (the coordinate system origin) to a specific georeference position on site (LLA-latitude, longitude and altitude). This scene is exported as a channel by uploading this content from local server (WampServer) to the delivery channel (Metaio cloud). The result of this deployment process is a free Metaio cloud app Junaio. The Junaio Channel acts as a website or a TV channel simulating a distributions platform or a browser for the AR experience. This channel goes through a validation process when it is first created. 

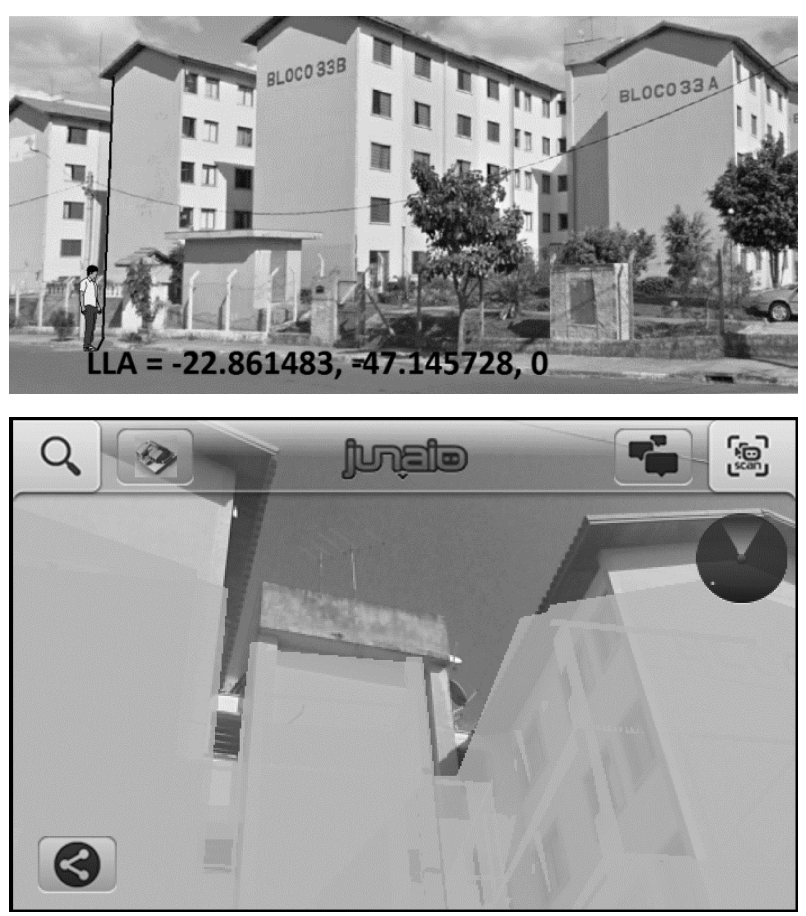

Fig. 8 Defined POI (point of interest) (with LLA) in the site and first results of real scale AR model visualization.

The created Junaio Channel for the POE of the Campinas $\mathrm{F}$ was tested and resulted in the images presented in Fig. 8. This action is equivalent to initial tests of the experiment, still presenting unsatisfactory results. Two difficulties still remain: controlled process for model scaling and strategy for best choice of system coordinate origin definition depending on observer position on site (Fig. 8).

\section{Conclusions}

The incorporation of AR in POE using out of the shelf applications is still difficult and with shortcomings. This imposes restriction to the long-term aim of improving retrofit identification while walking through the existing built environment. Table 1 resumes encountered difficulties.

The selected mobile AR development platform is complete and robust but in constant evolution and requires medium to high knowledge of programming. Modeling knowledge as technical information on treatment and optimization for virtual models for effective use in these systems is necessary. There is also an issue of limited levels of use with restricted
Table 1 Difficulties related to POE instrument incorporating AR.

\begin{tabular}{lll}
\hline $\begin{array}{l}\text { Type of } \\
\text { difficulty }\end{array}$ & $\begin{array}{l}\text { Mobile AR out of the } \\
\text { shelf solution }\end{array}$ & Modeling \\
\hline & $\begin{array}{l}\text { Medium to high } \\
\text { knowledge of } \\
\text { programming }\end{array}$ & $\begin{array}{l}\text { Medium to high } \\
\text { knowledge of model } \\
\text { development and } \\
\text { treatment }\end{array}$ \\
Description & $\begin{array}{l}\text { Positioning and scaling } \\
\text { of real scale model }\end{array}$ & $\begin{array}{l}\text { Low interoperability } \\
\text { between application } \\
\text { Balance between } \\
\text { simplicity and } \\
\text { representativeness }\end{array}$ \\
\hline
\end{tabular}

support, maintenance and installation associated to free use resources.

Questions of positioning and scaling of virtual objects in the real environment are important factors for mobile AR user in AECO. With mobile devices, calculations and geolocation are made directly by the device via GPS and digital compass, requiring no user intervention. However, tests indicate that it is possible that the available algorithms are limited, restricting movements to a specific situation as rotating around a point for example. This is inferred from the difficulty in maintaining the $3 \mathrm{D}$ content static while walking in one direction, but not if rotating around a fixed point. There was also difficulty in adjusting the $3 \mathrm{D}$ content scale in order to obtain 1:1 scale to site visualization. The unit used in the modeling application (that is $\mathrm{mm}$ or $\mathrm{m}$ in ArchiCAD, Revit or Sketchup) influenced this process. The choice of system coordinate origin in the $3 \mathrm{D}$ content (or BIM model) also influences visualization on site limiting/disturbing scale adjustments (small movements may take $3 \mathrm{D}$ content out of site with difficult recovering).

Still with respect to out of the shelf technology available for mobile $\mathrm{AR}$, the fact that the $3 \mathrm{D}$ content must be uploaded and rendered in real time though internet represents the need and dependence of good connection favouring the download time of information. However, in POE studies, the 3D models are in large scale and need to be detailed for precise comparison. BIM models for facility management, representing building use situation, are of the highest level of detail (LoD 500). Therefore, it seems to be 
counter intuitive to simplify 3D content for upload and download performance issues. In order not to cause impact of LoD on use of AR, a balanced point between simplicity and representativeness should be found in this case. This was the solution adopted in this experiment. The $\mathrm{LoD}$ of the model itself did not change, for only information that did not interfere with the verification of exterior renovations filtered from the model (as window glasses, internal equipments and so on).

In terms of modeling precautions, it should be observed that the original BIM model must represent, at least, final state of construction (when it was delivered to the user). This was not the case of the BIM model used, the design drawings did not include detailed information of external stairs, ramps, trash disposal, ordinances and water inlet. Thus, it should be noted that the model to be used in POE with AR has to contain all these original details to allow a correct analysis by comparing the content viewed.

\section{References}

[1] R.T. Azuma, A survey of augmented reality, presence: Teleoperators and virtual environments, Columbus [Online] 6 (4) (1997) 355-85, http://www.cs.unc.edu (accessed Apr. 20, 2010).

[2] W. Höhl, Interactive Environments with Open-Source-Software, 3D Walkthroughs and Augmented Reality for Architects with Blender 2.43, DART 3.0 and ARToolKit 2.72, Springer Wien New York, Vienna, 2009.

[3] G.C. Burdea, P. Coiffet, Virtual Reality Technology, 2nd ed., John Wiley \& Sons Inc., New Jersey, 2003.

[4] R.R. Amim, Augmented Reality Applied to Architecture and Urban Planning, Rio de Janeiro, Dissertation. Universidade Federal do Rio de Janeiro, 2007.

[5] W.F.E. Preiser, H.Z. Rabinowitz, E. White, Post-Occupancy Evaluation, Van Nostrand Reinhold, New York, 1988.

[6] D.C.C.K. Kowaltowski, A house design assistance program for the self-building process of the region of
Campinas, Brazil: Evaluation through a case study, Habitat International 29 (1) (2005) 95-111.

[7] T.H. Höllerer, S.K. Feiner, Mobile augmented reality, in: H. Karimi, A. Hammad (Eds.), Telegeoinformatics: Location-Based Computing and Services, Taylor and Francis Books Ltd., London, 2004.

[8] B. Thomas, W. Piekarski, B. Gunther, Using Augmented Reality to Visualize Architecture Design in an Outdoor Environment, in: Design Computing on the Net: DCNET, Sidney, 1999, http://citeseerx.ist.psu.edu (accessed Mar. 5, 2010).

[9] A. Rösler, Augmented Reality Games on the iPhone: What Are Some of the Possibilities and Problems Associated with the Creation of Augmented Reality Games for the Iphone?, Monography, Blekinge Institute of Technology, Karlskrona, 2009.

[10] O. Bimber, R. Raskar, Spatial Augmented Reality: Merging Real and Virtual Worlds, A. K. Peters, Ltd., Wellesley, 2005.

[11] A.R.M. Cuperschmid, M.R. Freitas, R.C. Ruschel, Technologies That Support Augmented Reality Applied in Architecture and Construction, Cadernos do PROARQ (Universidade Federal do Rio de Janeiro), 2012, http://www.proarq.fau.ufrj.br/revista/public/docs/Proarq1 9_TechnologiesReality_CuperschmidRuschelFreitas.pdf (accessed July 22, 2014).

[12] B. Succar, Building information modelling framework: A research and delivery foundation for industry stakeholders, automation in construction, Elsevier Science 18 (2009) 357-375.

[13] C. VEastman, P. Teicholz, R. Sacks, K. Liston, BIM Handbook: A guide to Building Information Modeling for Owners, Managers, Designers, Engineers and Contractors, John Wiley \& Sons, Hoboken, New Jersey, 2008.

[14] J. Bedrick, Organizing the Development of a Building Information Model, AECbytes, 2008, http://www.aecbytes.com/feature/2008/MPSforBIM.html (accessed May 28, 2013).

[15] American Institute of Architects Document G202 Project Building Information Modeling Protocol Form, California, AIA, 2013, http://www.aia.org/aiaucmp/groups/aia/documents/pdf/a iab099086.pdf (accessed July 22, 2014).

[16] F. Leite, A. Akcamete, B. Akinci, G. Atasoy, S. Kiziltas, Analysis of modeling effort and impact of different levels of detail in building information models, Automation in Construction 20 (2011) 601-609. 